\title{
Ultrastructural Analysis Reveals CAMP-Dependent Enhancement of Microvascular Endothelial Barrier Functions via Rac1-Mediated Reorganization of Intercellular Junctions
}

\author{
Volker Spindler, ${ }^{*}$ Dominik Peter, ${ }^{*}$ \\ Gregory S. Harms, ${ }^{\dagger}$ Esther Asan, ${ }^{*}$ \\ and Jens Waschke*
}

From the Institute of Anatomy and Cell Biology* and the RudolfVirchow-Center, ${ }^{\dagger}$ University of Würzburg, Würzburg, Germany

Evidence exists that cAMP stabilizes the endothelial barrier, in part via activation of the small GTPase Rac1. However, despite the high medical relevance of this signaling pathway, the mechanistic effects on intercellular contacts on the ultrastructural level are largely unknown. In microvascular endothelial cell monolayers, in which increased cAMP strengthened barrier properties, similar to intact microvessels in vivo, both forskolin and rolipram $(\mathrm{F} / \mathrm{R})$ to increase CAMP and 8-(4-chlorophenylthio)-2'-O-methyladenosine-3',5'-cyclic monophosphorothioate (O-Me-cAMP) to stimulate exchange protein directly activated by CAMP/ Ras proximate-1 (EPac/Rap 1) signaling enhanced transendothelial electrical resistance and induced activation of Rac1. Concurrently, augmented immunofluorescence intensity and linearization of signals at cell borders were observed for intercellular junction proteins VE-cadherin and claudin 5. Ultrastructural analysis of the intercellular contact zone architecture documented that exposure to F/R or O-Me-cAMP led to a significant increase in the proportion of contact sites displaying complex interdigitations of cell borders, in which membranes of neighboring cells were closely apposed over comparatively long distances; in addition, they were stabilized by numerous intercellular junctions. Interference with Rac1 activation by NSC-23766 completely abolished both barrier stabilization and contact zone reorganization in response to O-MeCAMP, whereas F/R-mediated Rac1 activation and barrier enhancement were not affected by NSC-23766. In parallel experiments using macrovascular endothelium, increased CAMP failed to induce Rac1 activation, barrier enhancement, and contact zone reorganization. These results indicate that, in microvascular endothelium, Rac1-mediated alterations in contact zone architecture contribute to
CAMP-induced barrier stabilization. (Am J Pathol 2011, 178:2424-2436; DOI: 10.1016/j.ajpath.2011.01.014)

The barrier between blood and the surrounding interstitium, limiting the exchange of fluid and solutes, is provided by a single layer of endothelial cells that are coupled by intercellular junctions. Various pathological conditions in humans, such as edema, hemorrhagic stroke, and vascular malformations, have been, at least in part, caused by impaired endothelial barrier function and disruption of cell junctions. ${ }^{1}$ In particular, edema formation in acute inflammation and sepsis is caused by the formation of intercellular gaps between neighboring endothelial cells, leading to increased paracellular permeability, a phenomenon that is largely confined to postcapillary venules. ${ }^{2,3}$ Because it is known that endothelial adherens junctions initiate cell contact formation and are required for the maintenance of cell junctions and tight junction control permeability by sealing the intercellular cleft, ${ }^{1}$ many studies investigating endothelial barrier functions have focused on the regulation of these junctions. Mechanisms involved in such regulatory processes are the turnover of junction proteins and their anchorage to the cytoskeleton. ${ }^{4}$ Claudin 5 , the typical transmembrane adhesion molecule of endothelial tight junctions, and vascular endothelial (VE)-cadherin, the endothelial integral membrane protein of adherens junctions, are coupled to the cortical actin cytoskeleton via adaptor proteins of the zonula occludens or catenin family, respectively. ${ }^{5}$

Supported by a grant from the Deutsche Forschungsgemeinschaft (Sonderforschungsbereich 688, Teilprojekt A4).

V.S. and D.P. contributed equally to this work.

Accepted for publication January 25, 2011

Supplemental material for this article can be found at $h t t p: / / a j p$ amjpathol.org or at doi: 10.1016/j.ajpath.2011.01.014.

Address reprint request to Jens Waschke, M.D., Institute of Anatomy and Cell Biology, University of Würzburg, Koellikerstrasse 6, 97070 Würzburg, Germany. E-mail: jens.waschke@mail.uni-wuerzburg.de. 
Signaling pathways involving the second messenger CAMP have most effectively mediated the reduction of microvascular paracellular permeability in vivo under both resting conditions and challenge with inflammatory mediators. ${ }^{2}$ Although several different mechanisms may be involved in cAMP-mediated stabilization of endothelial barrier properties, the regulation of cell junctions appears to be central. In this context, cAMP signaling mediated barrierprotective effects, at least in part via control of small GTPases, such as Rho GTPase family members RhoA and Rac1 or Rap 1. ${ }^{6,7}$ In particular, cAMP caused activation of Rac1 via both protein kinase $A$ and Epac-triggered activation of Rap $1 .^{8-10}$ More recently, CAMP- and cGMP-mediated Rac1 activation and endothelial permeability reduction have been dependent on the actin-binding vasodilatorstimulated phosphoprotein. ${ }^{11}$ Several barrier-disrupting mediators, such as tumor necrosis factor $\alpha$ and thrombin, and the bacterial endotoxin lipopolysaccharide reduced endothelial CAMP levels and inactivated this pathway ${ }^{12-15}$; some barrier-protective mediators are known to increase cAMP. ${ }^{6}$ These studies provided sound biochemical, light microscopic morphological, and functional evidence for cAMP as a central hub in the control of microvascular permeability. However, the interesting questions of whether and how cAMP-mediated effects on barrier properties are reflected in changes of structural properties of intercellular contact zones have not been adequately addressed.

Therefore, the present study was designed to characterize the effects of CAMP/Rac1 signaling on endothelial intercellular contact morphological features on the ultrastructural level in cultured microvascular and macrovascular endothelial cells by transmission electron microscopy (EM) and to correlate them with measurements of microvascular permeability both in vivo and in vitro.

\section{Materials and Methods}

\section{Cell Culture}

As previously described, ${ }^{16}$ primary human dermal microvascular endothelial cells (HDMECs; PromoCell, Heidelberg, Germany) were grown on uncoated cell culture flasks in endothelial growth medium MV containing supplement mix (PromoCell) in a humidified atmosphere with $5 \% \mathrm{CO}_{2}$. Cells were passaged using a kit (Detach kit-30; PromoCell) and used between passages 2 and 5. Immortalized microvascular mouse myocardial endothelial cells (MyEnd) $)^{17,18}$ were cultured on collagen 1-coated culture flasks and maintained in Dulbecco's modified Eagle's medium (SigmaAldrich, Munich, Germany) containing 10\% fetal calf serum and $50 \mathrm{U} / \mathrm{mL}$ penicillin/50 $\mu \mathrm{g}$ streptomycin. Primary porcine pulmonary artery endothelial cells (PAECs) were isolated by collagenase A treatment, as described elsewhere. ${ }^{19}$ Cells were cultured on collagen 1-coated culture flasks in medium 199 (M199; Sigma-Aldrich) supplemented with 10\% fetal calf serum (Biochrom, Berlin, Germany), $50 \mathrm{U} / \mathrm{mL}$ penicillin (Sigma-Aldrich), and $50 \mu \mathrm{g}$ streptomycin (Sigma-Aldrich) in a humidified atmosphere. The PAECs were used in passages 2 and 3 .
HDMECs were characterized by immunofluorescence with respect to their vascular or lymphatic origin (see Supplemental Figure S1 at $h$ ttp://ajp.amjpathol.org) to rule out extensive contamination with lymphatic endothelial cells. All cultured cells were membrane positive for the following markers of blood vessel origin: vascular endothelial growth factor receptor 2, angiopoietin receptor TIE-2, and platelet endothelial cell adhesion molecule 1. All cells were negative for lymphatic marker prospero homeobox protein 1; however, some (approximately 5\% to $10 \%$ ) of the cells displayed predominantly intracellular staining for lymphatic vessel endothelial hyaluronan receptor 1 .

\section{Reagents}

NSC-23766 (Calbiochem/Merck, Darmstadt, Germany) inhibits activation of Rac 1 by blocking the binding of the Rac1-specific GTP-exchange factors triple functional domain protein (Trio) and T-cell lymphoma invasion and metastasis 1 (Tiam1). ${ }^{20}$ NSC-23766 was used at $200 \mu \mathrm{mol} / \mathrm{L}$ for 30 minutes. To increase intracellular cAMP levels, we applied forskolin and rolipram (F/R) (both from Sigma-Aldrich) for 60 minutes at concentrations of $5 \mu \mathrm{mol} / \mathrm{L}$ and 10 $\mu \mathrm{mol} / \mathrm{L}$, respectively; and used 8-pCPT-2'-O-methyl-cAMP (O-Me-cAMP) (Biolog, Bremen, Germany) at $200 \mu \mathrm{mol} / \mathrm{L}$ for 60 minutes to activate predominantly Epac/Rap1 signaling.

\section{Animal Preparation and Hydraulic Conductivity Measurement}

Male and female rats were kept under conditions that conformed to the National Institutes of Health Guide for the Care and Use of Laboratory Animals, approved by the Regierung von Unterfranken. Wistar rats with a body weight ranging from $350 \mathrm{~g}$ to $450 \mathrm{~g}$ (Charles River Laboratories, Sulzfeld, Germany) were anesthetized with pentobarbital sodium (65 mg/kg body weight) and prepared as previously described. ${ }^{18}$ Briefly, the rat was placed on a heating pad with the mesentery pulled out and spread on a pillar after a midline incision. The mesentery was constantly suffused with Ringer's solution at $37^{\circ} \mathrm{C}$. A straight nonbranched segment of a mesenteric venular microvessel (diameter, 25 to $35 \mu \mathrm{m}$ ) was then cannulated with a glass micropipette and continuously perfused with Ringer's solution containing $10 \mathrm{mg} / \mathrm{mL}$ bovine serum albumin (BSA; Sigma-Aldrich) with or without F/R. As previously described, measurements of hydraulic conductivity $\left(L_{p}\right)$ were based on the modified Landis technique, which measures the volume flux per unit surface area across the wall of a perfused microvessel. ${ }^{2,18,21}$ Measurements were performed at approximately 10-minute intervals for up to 120 minutes. All animals were used for one experiment only.

\section{Measurement of Transendothelial Resistance}

An electric impedance sensing setup (ECIS 1600R; Applied BioPhysics Inc., Troy, NY) was used to measure the transendothelial resistance (TER) of endothelial monolayers, as previously described. ${ }^{16}$ HDMECs and PAECs were grown 
on an electrode array until reaching confluence after 8 to 10 days. After exchange to fresh media, baseline TER was measured for 15 minutes, followed by application of different mediators to each well of the array, as specified.

\section{Transmission EM}

Specimens for ultrastructural analyses of cultured cells were prepared as described by Wijsman and Shivers, ${ }^{22}$ with minor modifications. Confluent HDMEC and PAEC monolayers were fixed with $2.5 \%$ glutaraldehyde and $0.01 \%$ ruthenium red in $0.1 \mathrm{~mol} / \mathrm{L}$ sodium cacodylate buffer, $\mathrm{pH} 7.35$, for 1 hour at $4^{\circ} \mathrm{C}$. After rinsing in $0.1 \mathrm{~mol} / \mathrm{L} \mathrm{caco}$ dylate (three times for 5 minutes), cells were postfixed in $2 \%$ $\mathrm{OsO}_{4}$ in $0.1 \mathrm{~mol} / \mathrm{L}$ cacodylate for 1 hour at room temperature. Cells were rinsed (three times each for 5 minutes) again in cacodylate and dehydrated in ascending concentrations of methanol $(25 \%, 50 \%$, and $70 \% ; 10$ minutes each), placed for 1 hour in saturated uranyl acetate in $70 \%$ methanol in the dark, and then further dehydrated $(80 \%$, twice at $95 \%$, and twice at $100 \% ; 10$ minutes each). Thereafter, cells were equilibrated in ethoxy-propanol (twice for 10 minutes) and embedded in Epon 812. Vertically oriented semithin sections $(1 \mu \mathrm{m})$ were stained with methylene blue, and ultrathin sections were contrasted with uranyl acetate and lead citrate. ${ }^{23}$ Sections were analyzed and micrographs were obtained with an EM (LEO AB 912; Zeiss Smt, Oberkochen, Germany). Sections from at least three (PAECs) or four (HDMECs) independent cultures for each experimental condition were used for quantification, as specified in the Results section. Supplemental Table S1 (available at $h$ ttp://ajp.amjpathol.org) demonstrates the exact numbers of intercellular contact zones investigated for each experiment/condition.

Specimens from in situ microvessels were prepared from two rat mesenteries. Vessels were recannulated after a 60minute perfusion period with Ringer's/BSA solution and perfused with Ringer's solution containing $10 \mathrm{mg} / \mathrm{mL}$ lanthanum nitrate (Carl Roth $\mathrm{GmbH}$, Karlsruhe, Germany) for 20 seconds, according to a protocol from Adamson and Michel. ${ }^{24}$ Afterward, mesentery was excised, immersion fixed (for 5 hours at $4^{\circ} \mathrm{C}$ ), and further processed for embedding in Epon, as previously described for cultured cells. Ultrathin sections across the vessel diameter, downstream of the cannulation site, were examined after checking for the intraluminal presence of the electron-dense lanthanum nitrate.

\section{Cytochemistry}

Cells were grown to confluence on uncoated (HDMEC) or collagen 1-coated (PAEC) glass coverslips for 8 to 10 days. After incubation with mediators under various conditions, as previously outlined, culture medium was removed and monolayers were fixed and processed for immunostaining, as described elsewhere. ${ }^{14}$ Primary antibodies used (1:100 in PBS each) were goat anti-human VE-cadherin (Santa Cruz, Heidelberg, Germany), mouse anti-human claudin 5 (Invitrogen, Darmstadt, Germany), rabbit anti-human vascular endothelial growth factor receptor 2 (Abcam Plc, Cambridge, UK), mouse anti-human Tie-2 (Ab- cam Plc), mouse anti-human platelet endothelial cell adhesion molecule 1 (New England Biolabs, Frankfurt, Germany), rabbit anti-human lymphatic vessel endothelial hyaluronan receptor 1 (Abcam Plc), and rabbit anti-human prospero homeobox protein 1 (Abcam Plc). Alexa488-conjugated phalloidin was applied in a 1:60 dilution in PBS to label actin filaments. Monolayers were photographed with a confocal microscope (LSM 510; Carl Zeiss Microimaging Inc., Göttingen, Germany) with the same settings using a $\times 63$ numerical aperture 1.4 oil objective. Quantification of VE-cadherin and claudin 5 distribution was performed using computer software (Image J). For distribution, a rectangular marquee of $20 \times$ $5 \mu \mathrm{m}$ was drawn over more than 20 randomly chosen areas of HDMEC plasma membrane of at least three independent experiments; the mean intensity distribution of VE-cadherin and claudin 5 staining was recorded in each marquee. Values higher than a threshold of 20 arbitrary units (AUs) represented specific membrane staining. Thus, the mean width of membrane staining was calculated by the distance between the two intersection points of the intensity graph with a horizontal line drawn at $20 \mathrm{AUs}$.

For in situ immunofluorescence studies, rat mesenteric venules were perfused with Ringer's/BSA solution with or without $F / R$. Afterward, mesentery was fixed by suffusing the cannulation area with $2 \%$ paraformaldehyde in PBS, excised, and immunostained for VE-cadherin, as previously described. Venules were imaged with a confocal microscope (Leica SP5) using an X63 PL APO NA 1.20 water-immersion objective (both from Leica, Wetzlar and Mannheim, Germany). To cover the entire vessel, Z-stacks were performed with a step size of $1 \mu \mathrm{m}$ (approximately 25 images per vessel). Images were superposed using software (ImageJ).

\section{cAMP Detection and Activation Assays}

An enzyme-linked immunosorbent assay (ELISA)-based detection kit (Sigma-Aldrich) was applied to measure cellular cAMP levels following manufacturer's instructions. As previously described, ${ }^{16}$ commercially available kits (G-Lisa Activation Assays Biochem Kits; Cytoskeleton Inc., Denver, CO) were used for measurement of Rac1 and Cdc42 activity, according to manufacturer's recommendations. Signals were detected at $490 \mathrm{~nm}$ using a microplate spectrophotometer (Sunrise; Tecan GmbH, Crailsheim, Germany).

\section{Statistics}

Values throughout are expressed as mean \pm SE. Possible differences were assessed using the nonparametric Mann-Whitney rank sum test. Statistical significance was assumed for $P<0.05$.

\section{Results}

\section{CAMP-Mediated Barrier Enhancement in Vivo Was Paralleled by Linearization of VE-Cadherin Distribution}

Endothelial barrier properties in vivo were quantified by measuring the $L_{p}$ of single perfused rat postcapillary 

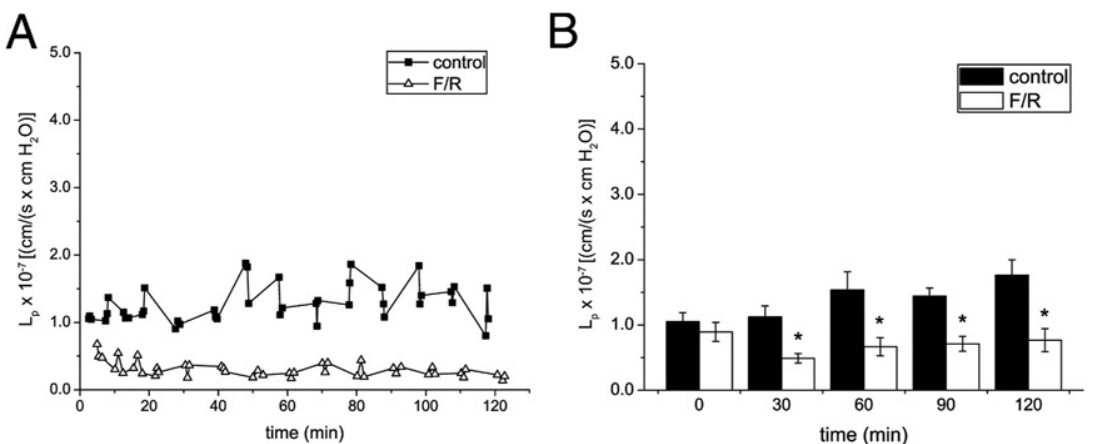

C
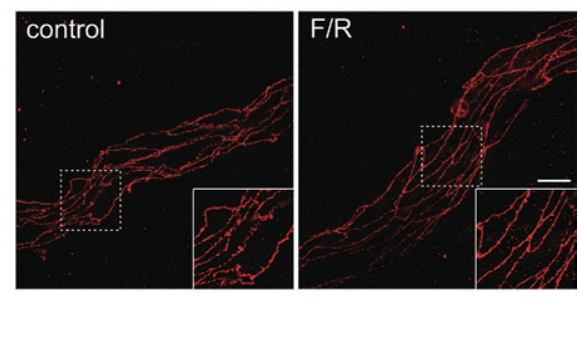

Figure 1. A and B: cAMP signaling enhanced endothelial barrier properties in vivo. The $\mathrm{L}_{\mathrm{p}}$ of intact rat mesenteric postcapillary venules was monitored over time. Adenylate cyclase activation by $\mathrm{F}$ and phosphodiesterase IV inhibition with $\mathrm{R}$ reduced $\mathrm{L}_{\mathrm{p}}$ beginning at 30 minutes compared with controls perfused with Ringer's/BSA solution only. A: One representative experiment. B: The results of six independent measurements for each condition. ${ }^{*} P<0.05$ versus the respective point of control perfusion. C: VE-cadherin immunostaining to delineate adherens junctions. Compared with controls, VE-cadherin appeared linearized at cell borders in F/R-perfused vessels. Insets: Magnifications $(\times 2)$ of the region within the dashed box. Representatives of four independent experiments are shown. Scale bar $=20 \mu \mathrm{m}$.

venules (Figure 1). Initial $L_{p}$ values of control microvessels perfused with Ringer's/BSA solution only were comparable to those of microvessels perfused with Ringer's/ BSA solution containing $10 \mu \mathrm{mol} / \mathrm{L}$ adenylate cyclase activator forskolin and $5 \mu \mathrm{mol} / \mathrm{L}$ phosphodiesterase IV inhibitor rolipram (F/R) to increase cAMP levels (1.05 \pm $0.14 \times 10^{7}\left[\mathrm{~cm} /\left(\mathrm{s} \times \mathrm{cm} \mathrm{H}_{2} \mathrm{O}\right)\right]$ and $0.90 \pm 0.15 \times 10^{7}$ $\left[\mathrm{cm} /\left(\mathrm{s} \times \mathrm{cm} \mathrm{H}_{2} \mathrm{O}\right)\right]$, respectively). Beginning at $30 \mathrm{~min}-$ utes, the $L_{p}$ of $F / R$-perfused vessels was significantly lower compared with that of controls (30 minutes, $0.50 \pm$ $0.07 \times 10^{7}\left[\mathrm{~cm} /\left(\mathrm{s} \times \mathrm{cm} \mathrm{H}_{2} \mathrm{O}\right)\right]$ versus $1.12 \pm 0.17 \times 10^{7}$ $\left[\mathrm{cm} /\left(\mathrm{s} \times \mathrm{cm} \mathrm{H}_{2} \mathrm{O}\right)\right] ; 120$ minutes, $0.77 \pm 0.18 \times 10^{7}$ $\left[\mathrm{cm} /\left(\mathrm{s} \times \mathrm{cm} \mathrm{H}_{2} \mathrm{O}\right)\right]$ versus $1.76 \pm 0.24 \times 10^{7}[\mathrm{~cm} /(\mathrm{s} \times$ $\mathrm{cm} \mathrm{H}_{2} \mathrm{O}$ )] in controls).

To investigate whether changes in $L_{p}$ were paralleled by morphological changes, microvessels were fixed in situ after control or F/R perfusion and stained for VEcadherin (Figure 1C). Control microvessels displayed membrane-bound VE-cadherin distribution that, in some parts, appeared fine and frayed. In F/R-perfused vessels, VE-cadherin immunostaining was augmented and typically linearized along cell borders compared with controls. These data demonstrate that CAMP increased barrier properties of intact microvessels, which was accompanied by linearization of VE-cadherin staining.

\section{CAMP Enhances Endothelial Barrier Properties in Rac1-Dependent Manner in Microvascular but Not in Macrovascular Endothelial Cells}

To further investigate the effects of a cAMP increase on endothelial barrier properties, we used cultured endothelial cells. HDMECs were grown on gold electrode arrays, and TER was measured to assess endothelial barrier functions (Figure 2A). The addition of F/R increased TER to values of $155 \% \pm 6 \%$ of baseline after 60 minutes; for control conditions, no change was detectable $(100 \% \pm$ $6 \%$ ). Similarly, predominant activation of the Epac/Rap1 pathway with CAMP analogue O-Me-cAMP increased TER to $123 \% \pm 6 \%$. Under both F/R and O-Me-cAMP treatment, TER remained continuously elevated for 160 minutes compared with controls.
It is well established that cAMP enhances endothelial barrier properties, at least in part by activation of RhoGTPase Rac1. ${ }^{6}$ To confirm the role of Rac1 in CAMPmediated barrier stabilization in HDMEC, we used NSC23766. This compound specifically interferes with the GTP-exchange factors Tiam1 and Trio and, thus, prevents activation of Rac1. Treatment of HDMECs with NSC-23766 (200 $\mu \mathrm{mol} / \mathrm{L})$ for 30 minutes reduced TER to $62 \% \pm 2 \%$ of baseline values, indicating that a constant activation of Rac1 is necessary to maintain endothelial barrier properties under resting conditions (Figure 2B). The addition of F/R to NSC-23766-pretreated HDMECs rescued barrier breakdown and increased TER to control levels after 60 minutes $(97 \% \pm 6 \%)$, whereas treatment with O-Me-cAMP failed to augment TER $(71 \% \pm 2 \%)$.

Interestingly, in cultured porcine macrovascular PAECs, F/R and O-Me-cAMP failed to increase TER (Figure 2C). Compared with controls ( $97 \% \pm 1 \%$ of baseline values after 60 minutes), cAMP increase by $F / R$ yielded values of $90 \% \pm$ $2 \%$ of baseline and the addition of O-Me-cAMP resulted in values of $100 \% \pm 1 \%$ after 60 minutes. To exclude that F/R under these conditions was ineffective to increase CAMP, we measured total cellular cAMP levels in HDMECs and PAECs (Figure 2D). The F/R (60 minutes) increased cAMP levels in both cell lines to $420 \% \pm 40 \%$ and $570 \% \pm 30 \%$ of untreated controls, respectively.

To investigate the contribution of Rac1 activation to barrier increase in response to CAMP elevation, we quantified changes of Rac1 activity by ELISA-based measurements (Figure 2E). In HDMECs, both F/R and O-MecAMP enhanced Rac1 activity (174\% $\pm 10 \%$ and $152 \% \pm 16 \%$ of controls, respectively) after 60 minutes. Pretreatment with NSC-23766 completely abolished OMe-cAMP-induced Rac1 activation $(110 \% \pm 7 \%)$ and blunted the effect of F/R on Rac1 activation to $126 \% \pm$ $17 \%$ of controls. Next, we measured the effect of F/Rmediated CAMP increase on Rac1 activity in PAECs that did not respond with barrier enhancement. Interestingly, in contrast to HDMECs, F/R did not augment Rac1 activity compared with controls in PAECs $(95 \% \pm 14 \%$ and $103 \% \pm 15 \%$, respectively), indicating that the different efficacy of cAMP in strengthening endothelial barrier prop- 
A

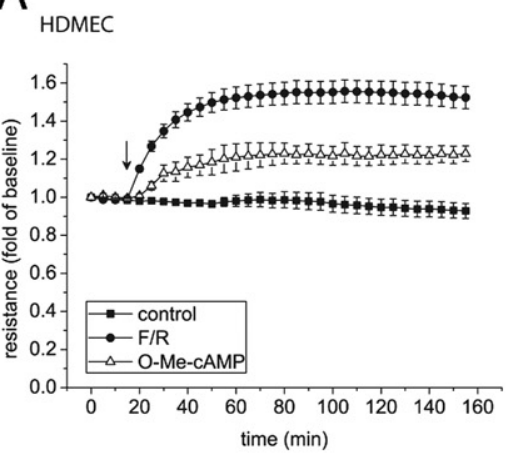

B

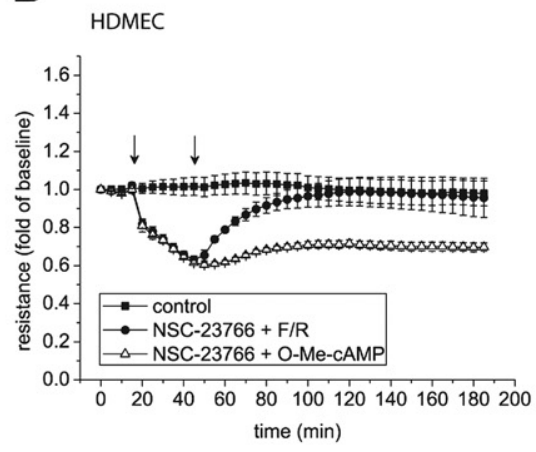

C PAEC

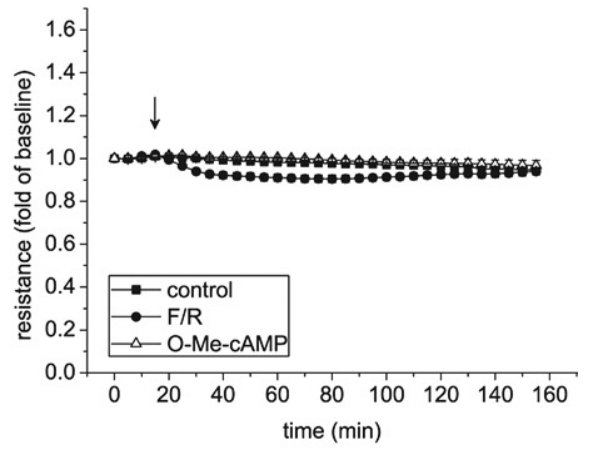

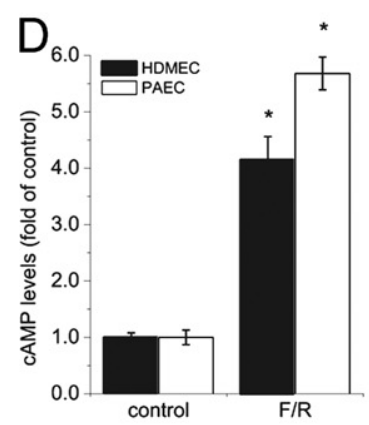
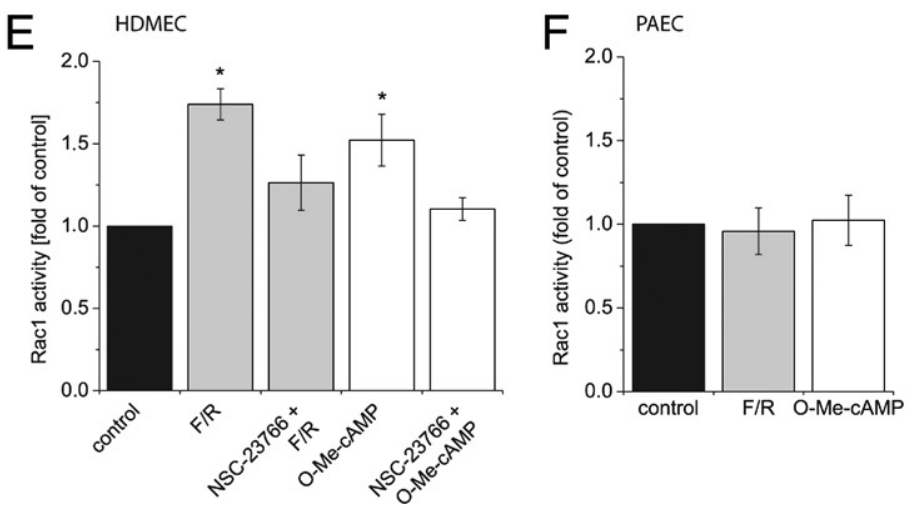

G

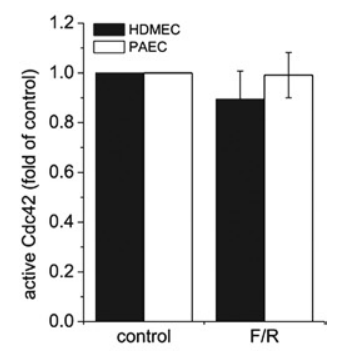

Figure 2. cAMP enhanced endothelial barrier properties in an Rac1-dependent manner in microvascular, but not in macrovascular, endothelial cell cultures. A: TER was increased in microvascular HDMECs by incubation with F/R or O-Me-cAMP to predominantly trigger Epac1/Rap1-specific signaling ( $n=6$, arrow indicates addition of F/R or O-Me-cAMP). B: Interference with Rac1 activity by NSC-23766 (30 minutes) reduced TER, which was restored by F/R but not by O-Me-cAMP ( $n=6$; first arrow indicates addition of NSC-23766, and second arrow indicates addition of F/R or O-Me-cAMP). C and D: No changes in macrovascular PAECs were detectable after treatment with F/R or O-Me-cAMP $(\mathbf{C}, n=6$, arrow indicates addition of F/R or O-Me-cAMP), although F/R effectively elevated cAMP levels after 60 minutes in both HDMECs and PAECs, as determined by ELISA-based cAMP measurements (D, $n=5)$. E: In HDMECs, both F/R and O-Me-cAMP (60 minutes) activated Rac1, which is blunted by preincubation with NSC-23766 (30 minutes, $n>4)$. F: In contrast, no Rac1 activation was inducible by F/R or O-Me-cAMP treatment in PAECs $(n=6)$. G: No changes in Cdc42 activity were detected after a cAMP increase with F/R in HDMECs or PAECs $(n=8) .{ }^{*} P<0.05$ compared with the respective control conditions.

erties in HDMECs and PAECs may have been caused by differences in Rac1 activation (Figure 2F). Cdc42 activity was not significantly changed in both HDMECs (99\% $\pm 9 \%)$ and PAECs $(90 \% \pm 11 \%)$ after incubation with F/R compared with controls (Figure 2G).

\section{cAMP-Induced Reorganization of Adherens and Tight Junctions Is, in Part, Rac1 Dependent}

Tight and adherens junctions are thought to form the molecular backbone of the endothelial barrier by regulating paracellular fluid flux. To visualize whether changes in these junctions correspond to alterations in TER, we performed immunostaining of adherens junction protein VEcadherin and tight junction molecule claudin 5 , together with phalloidin staining of actin filaments (F-actin) in both HDMECs and PAECs. Moreover, similar to a previous study, ${ }^{14}$ the staining intensity and width of immunofluorescent labeling at cell borders were used as parameters to quantitatively assess changes in adherens and tight junction protein distribution in response to cAMP signaling. In HDMECs, VE-cadherin and claudin 5 immunofluorescence were distributed linearly along cell borders and in short lines orthogonal to cell borders, presumably representing multiple lateral cell protrusions interdigitat- ing and forming tortuous cell contact zones in the $x-y$ plane under control conditions (Figure 3, A and B). ${ }^{25}$ The actin network was predominantly composed of cortical filaments, with few stress fibers visible in the cytoplasm (Figure 3C). After treatment with either F/R (Figure 3, D-F) or O-Me-cAMP (Figure 3, G-I), staining of both junctional proteins and F-actin appeared intensified at the cell membrane and laterally extended immunoreactive lines were markedly reduced. Quantification confirmed that VE-cadherin fluorescence increased to $177 \pm 6 \mathrm{AUs}(\mathrm{F} / \mathrm{R})$ and $176 \pm 5$ AUs (O-Me-cAMP) compared with $115 \pm 7$ AUs in controls, together with a reduced width of the fluorescence-containing zone, possibly representing a reduction in lateral interdigitations (F/R, $3.3 \pm 0.2 \mu \mathrm{m}$; O-MeCAMP, $4.8 \pm 0.5 \mu \mathrm{m}$; control, $8.3 \pm 0.6 \mu \mathrm{m})$. Similarly, claudin 5 staining was increased under $\mathrm{F} / \mathrm{R}$ and $\mathrm{O}-\mathrm{Me}$ cAMP treatment to $151 \pm 5 \mathrm{AUs}$ and $160 \pm 4 \mathrm{AUs}$, respectively, versus $118 \pm 6$ AUs in controls; and width was reduced $(2.4 \pm 0.2 \mu \mathrm{m}$ and $2.4 \pm 0.2 \mu \mathrm{m}$, respectively, versus $6.3 \pm 0.5 \mu \mathrm{m}$ in controls). The inhibition of Rac 1 activation by NSC-23766 treatment (Figure 3, J-L) resulted in pronounced stress fiber formation, and intercellular gaps were present. VE-cadherin immunofluorescence intensity and width were $120 \pm 9$ AUs and $6.8 \pm 0.4 \mu \mathrm{m}$, respectively; and quantification of claudin 5 staining re- 

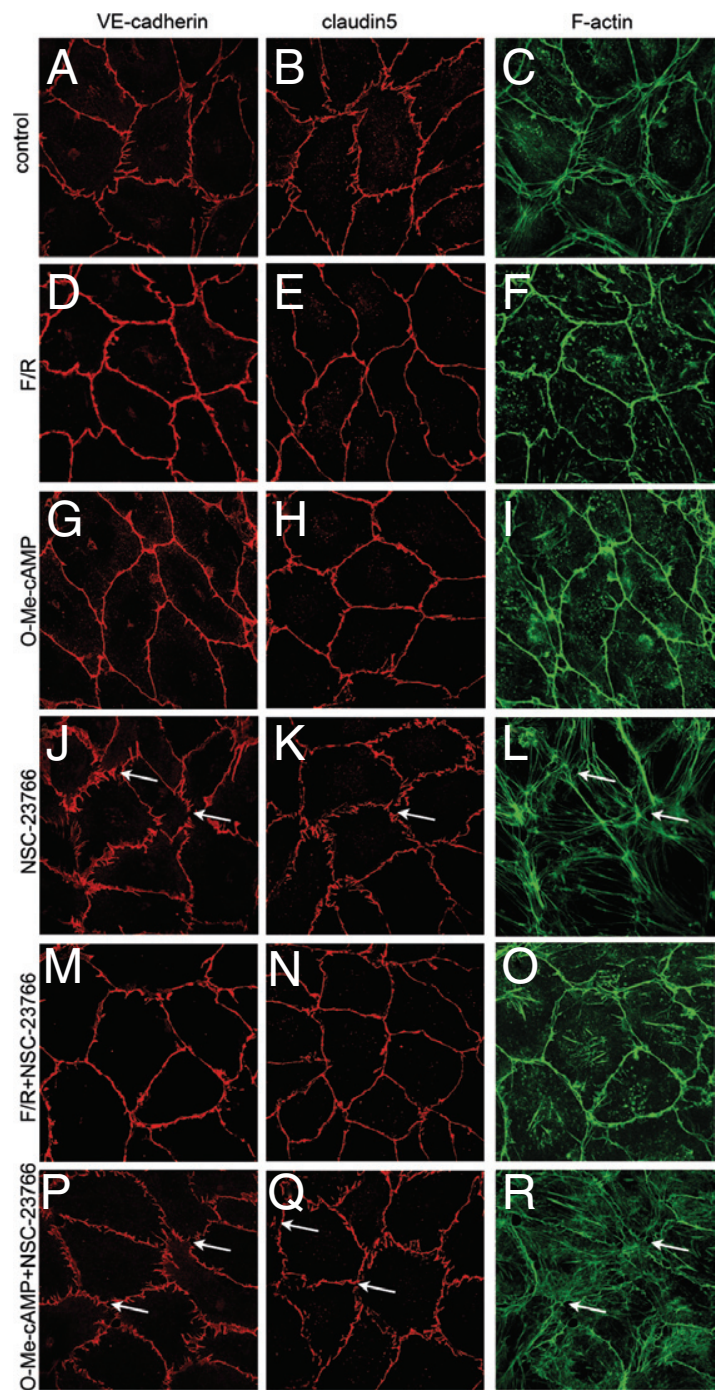

S

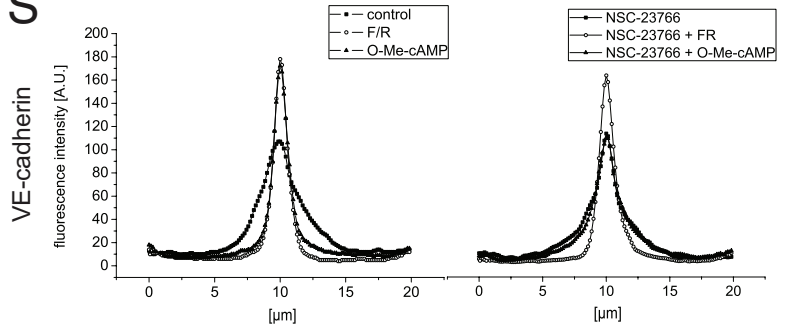

T
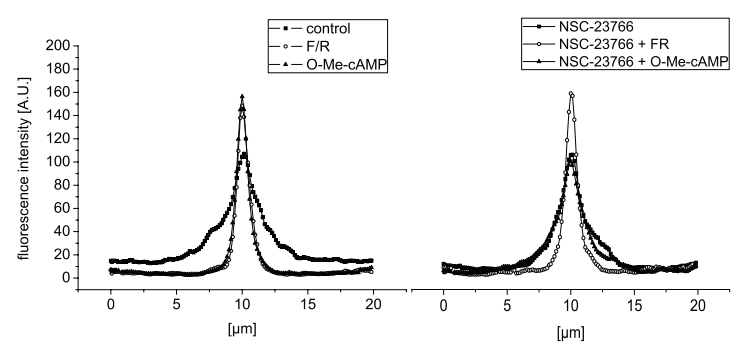

U
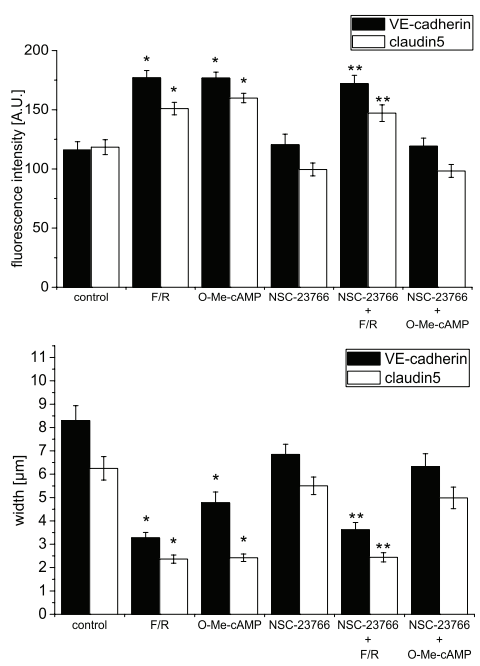

Figure 3. Reorganization of VE-cadherin and claudin 5 distribution by cAMP/Rac1 signaling in HDMECs. Compared with controls (A and B), incubation with F/R (D and $\mathbf{E}$ ) and O-Me-cAMP ( $\mathbf{G}$ and $\mathbf{H})$ for 60 minutes each intensified and narrowed the zone containing VE-cadherin and claudin 5 staining at cell borders. Cortical F-actin appeared enhanced after a cAMP increase (C, F, and I). NSC-23766 (30 minutes) induced intercellular stress fiber and gap formation (arrows, J-L), which was blocked by the addition of F/R (M-O) but not by O-Me-cAMP ( $\mathbf{P}-\mathbf{R}$, arrows point to intercellular gaps). Images are representatives of five independent experiments. Scale bar $=20 \mu \mathrm{m}$. Quantification of signal intensity distribution at cell borders ( $\mathbf{S}$ and $\mathbf{T})$ confirmed these results $(n>20)$. U: Mean intensity peak observed at cell borders. V: Width of VE-cadherin at a cutoff of 20 AUs of fluorescence intensity. ${ }^{*} P<0.05$ versus control, and ${ }^{* *} P<0.05$ versus NSC-23766 treatment.

vealed an intensity of $100 \pm 5$ AUs and a width $5.5 \pm 0.4$ $\mu \mathrm{m}$. Moreover, NSC-23766 blocked VE-cadherin and claudin 5 reorganization in response to O-Me-cAMP treatment (Figure 3, M-O; VE-cadherin intensity and width, $119 \pm 7$ AUs and $6.3 \pm 0.5 \mu \mathrm{m}$, respectively; claudin 5 intensity and width, $98 \pm 5 \mathrm{AUs}$ and $5.0 \pm 0.5 \mu \mathrm{m}$, respectively), whereas changes induced by $F / R$ incubation were not inhibited by interference with Rac1 activation (Figure 3, P-R; VE-cadherin intensity and width, $172 \pm 7$ AUs and $3.6 \pm 0.3 \mu \mathrm{m}$, respectively; claudin 5 intensity and width, $147 \pm 7 \mathrm{AUs}$ and $2.4 \pm 0.2 \mu \mathrm{m}$, respectively).

VE-cadherin and claudin 5 staining of PAECs demonstrated a similar distribution pattern as in HDMECs. However, in contrast to HDMECs, no pronounced changes in the distribution of VE-cadherin and claudin 5 were detectable in PAECs after F/R and O-Me-cAMP treatment, which was also confirmed by quantification (Figure 4).

\section{Reorganization of Intercellular Contact Zones in Response to cAMP Signaling on the Ultrastructural Level}

Because the ultrastructural changes of cell contact sites accompanying cAMP-induced enhancement of barrier properties are largely unknown, we performed transmission EM of vertical sections of confluent endothelial cell monolayers. Ultrastructural analysis showed that, under all experimental conditions, HDMECs grew as a single layer of flat cells. In contact zones of adjacent cells, most often, one cell contacted and/or overlapped another cell (Figure 5). Rarely, more than two cells overlapped (data not shown). Contact zones showed numerous regions of close apposition of contacting cell membranes, with intercellular clefts displaying uniform width (Figure 5). Occasionally, gap junctions of varying extent were seen 

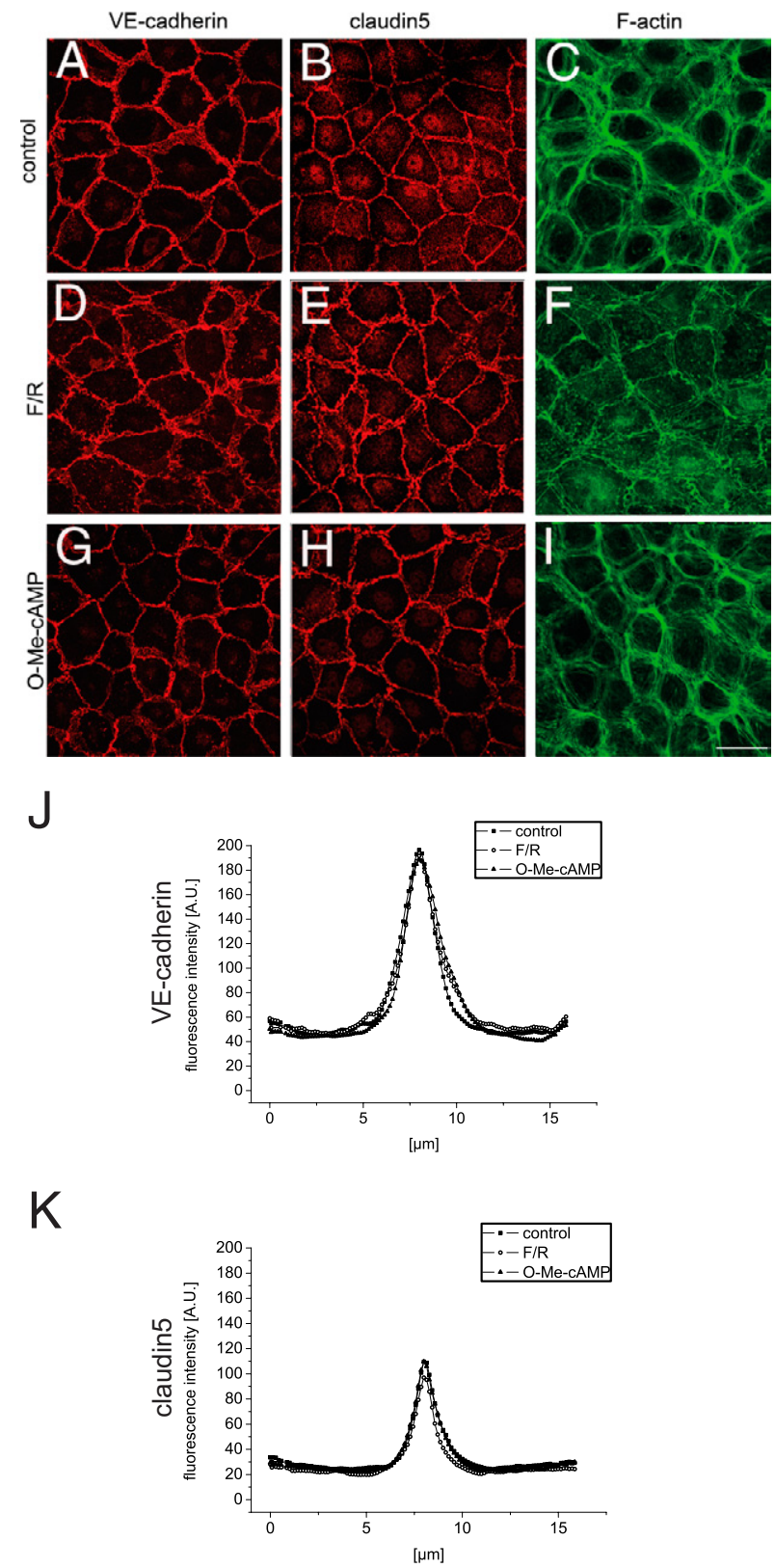

Figure 4. Reorganization of junctional molecules by cAMP/Rac1 signaling was not detected in PAECs. Similar to HDMECs, VE-cadherin and claudin 5 immunostaining localized to cell borders under controls $(\mathbf{A}-\mathbf{C})$. A reorganization of junctional molecules was not detectable after incubation with $\mathrm{F} / \mathrm{R}$ (D-F) or O-Me-cAMP (G-I). Stress fibers were largely reduced in response to F/R. Images are representatives of five independent experiments. Scale bar $=$ $20 \mu \mathrm{m}$. Quantification ( $\mathbf{J}$ and $\mathbf{K}$ ) confirmed the absence of changes of signal intensity and width at cell borders $(n>20)$.

(Figure 5A). More often, contacting cell processes formed tight and adherens junctions. Adherens junctions were defined as regions of contact zones in which the intercellular cleft appeared to contain more electrondense material than the adjacent intercellular spaces and in which plaquelike cytoplasmic accumulations of electron-dense material were observed on both sides of the apposing cell membranes (Figure 5B). Occasionally, microfilaments were associated with adherens junction plaques (data not shown). Frequently, apposed cell membranes in or near the adherens junction zones converged and, in some instances, fused (Figure 5C), indicating that tight junctions were intermingled with adherens junctions in these locations (Figure 5D). ${ }^{26,27}$ Taken together, ultrastructural features of HDMEC contact zones were generally in line with data from previous studies ${ }^{24,28-32}$ on microvascular endothelium in situ and in culture.

For comparative analysis of possible correlations between intercellular contact zone architecture and differential barrier properties, the morphological features of contact zones and the occurrence of junctions were studied and quantified under the different experimental conditions. Under control conditions, most contact zones between adjacent HDMECs were formed by one flat cell extension overlapping an equally flat extension of its neighbor. Obvious junctions were often absent at these contact sites (Figure 5H). In contrast, in HDMEC monolayers treated with F/R or O-Me-cAMP, a frequently observed morphological feature of cell contact zones consisted of two comparatively thicker extensions of adjacent cells interdigitating with several fingerlike protrusions extending one on top of the other from their lateral surfaces, leading to a complex and tortuous appearance of the intercellular cleft. Numerous buttonlike junctions stabilized the contact zone (Figure 5, F and G). In O-Me-cAMP-treated HDMEC monolayers preincubated with NSC-23766, most cell contact zones displayed a similar configuration, as in controls (Figure 5J). However, NSC-23766 treatment, followed by F/R incubation, resulted in cell contact zones dominated by the vertically orientated interdigitating appearance (Figure 5I).

To quantify these changes, we grouped the morphological characteristics of intercellular contact zones into four categories. Category 1 consisted of cell contact sites with an appearance as depicted in Figure $5 \mathrm{H}$ (ie, a simple overlap of two flat cell extensions of adjacent cell without junctions). Category 2 showed the same morphological characteristics and, in addition, contained junctions. In category 3, neighboring cells showed complex apicobasal interdigitations, but junctions were absent. Finally, category 4 displayed similar morphological characteristics as category 3 but bore visible junctions (Figure 5E). In total, for each condition, between 176 and 418 HDMEC intercellular contact zones were examined from at least four independent experiments. Supplemental Table S1 (available at http://ajp.amjpathol.org) delineates the exact numbers for each experiment.

As summarized in Figure $5 \mathrm{~K}$, for treatment with F/R and O-Me-cAMP, quantitative evaluation revealed that, in controls, categories 1 and 2 represent the fact that simple overlaps accounted for approximately $77 \%$ of all contact zones found; in F/R- and O-Me-cAMP-treated cultures, simple overlaps were found in only $53 \%$ and $54 \%$ of the contact zones, respectively. In particular, reciprocal changes of the occurrence of categories 1 and 4 were detected after treatment, whereas the frequency of categories 2 and 3 did not change compared with controls (Figure 5K). Thus, the effects were best described as a ratio $\left(R_{4 / 1}\right)$ of the percentage of category 4 complex contact zones/percentage of category 1 simple contact sites (Figure 5L). In controls, $\mathrm{R}_{4 / 1}$ was $0.6 \pm 0.2$. Treatment with F/R significantly increased $R_{4 / 1}$ by approximately eight- 
A
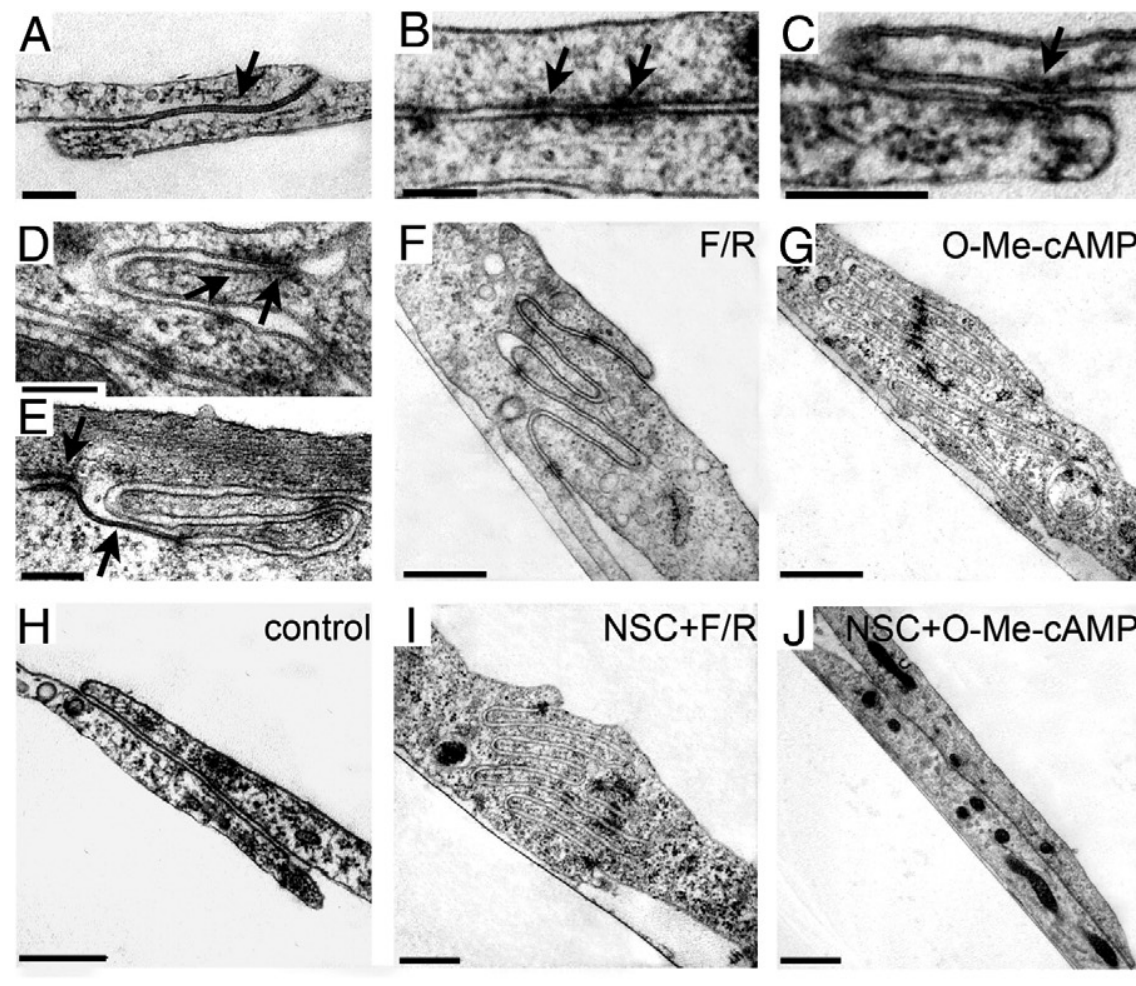

$\mathrm{K}$

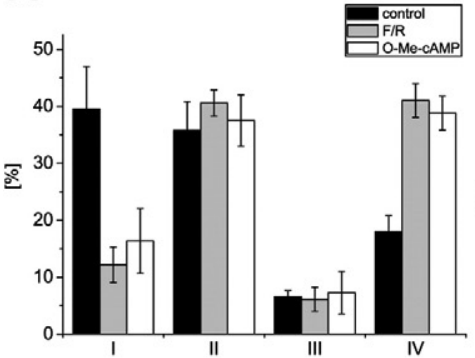

category

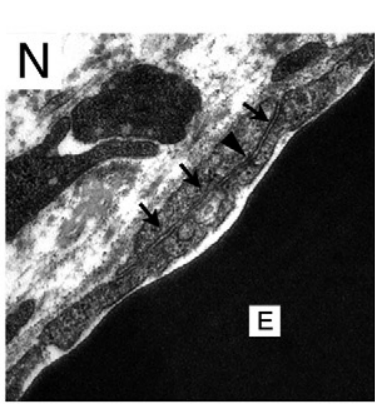

F/R

L

M
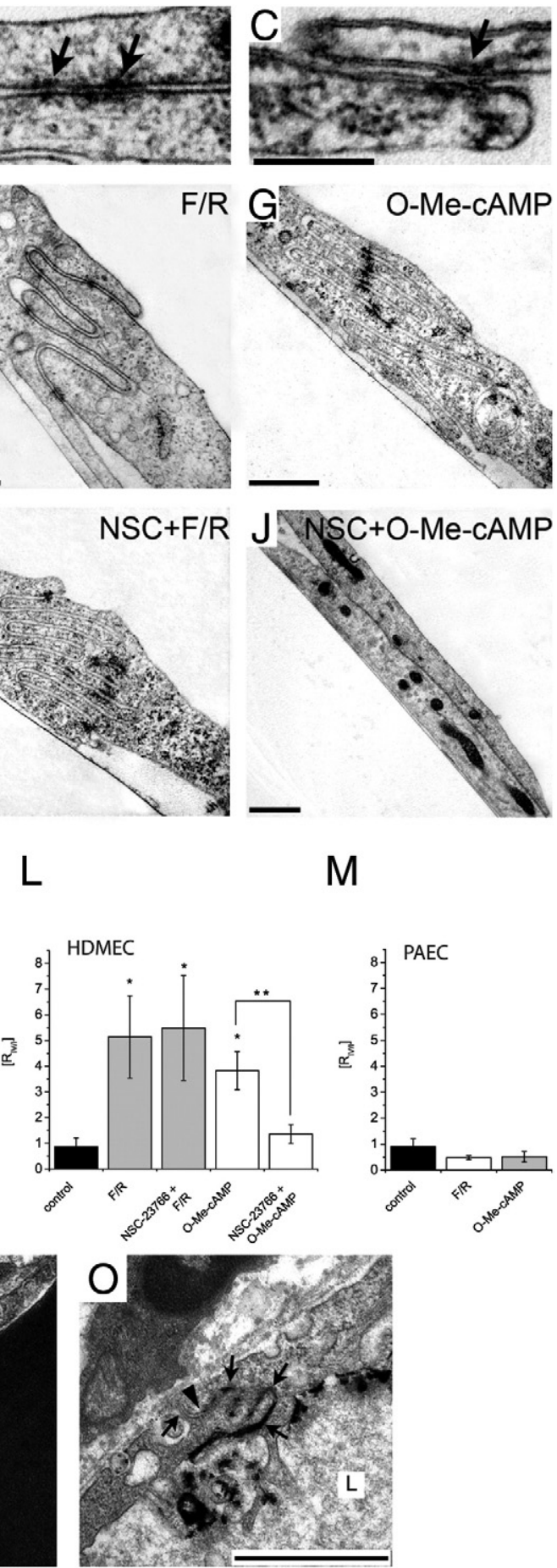

Figure 5. EM revealed prominent contact zone reorganization in response to cAMP-mediated Rac1 signaling in HDMECs. Vertically oriented sections of HDMEC (A-D and $\mathbf{F}-\mathbf{J})$ and $\operatorname{PAEC~(E)~}$ monolayers were examined by EM to investigate intercellular contact zones. Gap junctions (A arrow; $\mathbf{E}$, right arrow), adherens junctions (B arrows; E, left arrow), tight junctions $(\mathbf{C}$, arrow), and junctions composed of intermingled adherens (D, left arrow) and tight junctions (D right arrow) were detectable. In control $\mathrm{HD}$ MEC sections (H), most intercellular cell contact zones consisted of simple overlaps without junctions (category 1). In contrast, HDMEC contact sites displayed predominantly complex interdigitating morphological features with numerous junctions under conditions of increased cAMP/ Rac1 signaling (category 4; F and $\mathbf{G}$ ). Pretreatment with NSC-23766 prevented O-Me-cAMPinduced junction reorganization (J), whereas no changes were detected with $\mathrm{F} / \mathrm{R}(\mathbf{I})$. An analysis of the relative frequency of contact zones of different categories (described in the Results section) indicated treatment-induced changes in categories 1 and $4(\mathbf{K})$. F/R and O-Me-cAMP significantly increased $\mathrm{R}_{4 / 1}$. This increase was prevented by preincubation with NSC-23766 in case of O-MecAMP but not of $\mathrm{F} / \mathrm{R}(n=4$ to 6$)(\mathbf{L})$. In PAECs, no increase in $\mathrm{R}_{4 / 1}$ in response to increased cAMP signaling was detectable $(\mathbf{M}, n=3)$. $\mathbf{N}$ and $\mathbf{O}$ : In rat mesenteric postcapillary venules perfused under control conditions and labeled with lanthanum nitrate (black precipitate in $\mathbf{O}$ ), simple overlapping (N) and complex interdigitating $(\mathbf{O})$ intercellular contact zones were observed between endothelial cells, similar to contact zones found in HDMEC monolayers. Arrows delineate intercellular clefts, which in the right panel contain electron-dense lanthanum nitrate. Arrowheads depict a putative adherens junction in $\mathbf{N}$ and a putative tight junction stopping intercellular lanthanum diffusion in O. E indicates erythrocyte within the vessel lumen $\mathrm{L}$, vessel lumen. ${ }^{*} P>0.05$ versus control, and ${ }_{* * *} P>0.05$. Scale bars: $200 \mathrm{~nm}(\mathbf{A}-\mathbf{E}) ; 500 \mathrm{~nm}(\mathbf{F}-\mathbf{J})$ $1000 \mathrm{~nm}(\mathbf{N}$ and $\mathbf{O})$ fold to $5.1 \pm 1.6$. The F/R effect was not blocked by pretreatment with NSC-23766 (5.5 \pm 2.0$)$. Similar to F/R, O-Me-cAMP increased $R_{4 / 1}(3.8 \pm 0.7)$. However, preincubation with NSC-23766 blocked contact zone reorganization on the ultrastructural level, as revealed by $R_{4 / 1}$ $(1.3 \pm 0.4)$, which was not significantly different compared with controls. In summary, these data demonstrate that increased endothelial barrier function is accompanied by ultrastructural changes of intercellular contact zone architecture.
We also used a microvascular endothelial cell line derived from mouse myocardium (MyEnd) for some of the experiments (data not shown), which may be better comparable to the in vivo situation measured in rat mesentery. These cells displayed similar intercellular contact zone morphological features as HDMECs; however, the increase in the frequency of complex contact sites in response to cAMP elevation was less prominent. Accordingly, in TER experiments, monolayers (MyEnd) responded to F/R treatment with a mildly increased resistance only, which further indi- 
cates that the intercellular contact zone reorganization is a morphological correlate for increased barrier functions.

Cultured confluent PAEC monolayers displayed typical ultrastructural features of this endothelial cell type ${ }^{33}$ and formed single layers of comparatively fewer flat cells than HDMECs. In contact zones, one cell often extended a long flat process on top of a thicker extension of its neighbor. All types of intercellular junctions described for HDMECs were present (Figure 5E). Gap junctions appeared to be more frequent and larger than observed in HDMECs. Contact zone morphological characteristics were, in principle, similar to those described for microvascular endothelial cells, ranging from simple overlaps to complex intertwined cell contact zones. Quantification of 125 to 160 contact sites of three independent experiments (see Supplemental Table S1 at http://ajp.amjpathol.org) revealed a similar category $4 /$ category 1 ratio in the control macrovascular compared with control microvascular cells $(0.9 \pm 0.3$, Figure $5 \mathrm{M})$. However, in contrast to HDMECs, no significant changes in $\mathrm{R}_{4 / 1}$ were detectable in response to F/R or O-Me-CAMP $(0.5 \pm 0.1$ and $0.5 \pm 0.2$, respectively). Under these conditions, because no increase in barrier properties was detectable in PAECs (Figure 2C), these results point to a central role of cell contact site reorganization for increasing endothelial barrier properties.

Ultrastructural analysis of rat mesenteric microvessels under conditions of control perfusion was performed to compare the morphological characteristics of intercellular contact zones in vivo with the in vitro findings on microvascular endothelial cell contact sites. Reperfusion of cannulated vessels with a solution containing lanthanum nitrate allowed identification of perfused vessels. Intercellular clefts displaying both simple overlaps and complex interdigitations were observed in endothelia of identified vessels, similar to our findings in microvascular cell culture (Figure 5, $\mathrm{N}$ and $\mathrm{O}$ ). Although junctions were less clearly recognizable in vivo than in vitro, some small putative adherens junctions (Figure $5 \mathrm{~N}$ ) were found; and the fact that lanthanum nitrate diffused into intercellular clefts only for a limited distance (Figure 50) indicated that tight junctions obstructed its diffusion path. No evidence of lanthanum nitrate diffusion through the entire interendothelial cleft to the subendothelial space was observed at any point. Thus, refined studies will be necessary to visualize possible changes in permeability in response to a cAMP increase.

\section{Further Characterization of Intercellular Contact Zone Morphological Features}

Additional parameters of ultrastructural morphological characteristics were analyzed for the different contact zone categories in HDMECs to further compare the contact site architecture under the different experimental conditions and to detect possible correlations with the light microscopic and functional changes. As depicted in Figure 6D, we measured and compared structural properties of categories separately. We determined the cleft length (ie, the distance in which membranes of adjacent cells were apposed). Contact cleft lengths showed a wide variability, ranging from several hundred to more than $10,000 \mathrm{~nm}$. Comparison of categories 1 and 4 showed significantly longer intercellular cleft lengths in complex than in simple contact sites, irrespective of treatment; no significant changes in cleft lengths between treatment groups were detectable (Figure 6A). In addition, the mean cleft length of all complex contact zones taken together (categories 3 and 4) was substantially longer than that of simple contact zones (category 1 versus 2: $4493 \pm 245 \mathrm{~nm}$ versus $3219 \pm 132 \mathrm{~nm}$ ). Thus, because the proportion of complex contact zones was considerably higher in F/R-, NSC-23766/F/R-, and O-MecAMP-treated cultures than in controls and in NSC23766/O-Me-cAMP-treated endothelial cultures (increased $\mathrm{R}_{4 / 1}$, Figure $5 \mathrm{~K}$ ), the data indicate that the total cleft length is substantially increased in cultures displaying increased barrier properties.

Next, we evaluated the maximum width of cell overlap that varied in categories 1 and 4 from $1810 \pm 160 \mathrm{~nm}$ to $3360 \pm 800 \mathrm{~nm}$ under all experimental conditions (Figure $6 \mathrm{~B})$. Finally, we measured the maximum width of the junction-containing area of categories 2 and 4 that was in the range of $700 \mathrm{~nm}$ without prominent changes under the different conditions (Figure 6C). Taken together, these parameters showed a wide variability under the different experimental conditions but failed to reveal a specific pattern that may account for the light microscopically visible linearization of the immunolocalization of junction molecules.

\section{Discussion}

Intercellular junctions are known to be key players in the formation and maintenance of the endothelial barrier. Numerous investigations ${ }^{4,6}$ have been performed, and many signaling pathways have been documented to be involved in regulating endothelial barrier properties. In addition, ultrastructural changes in intercellular contact sites in response to barrier-compromising stimuli have been investigated, starting in the early 1960s. ${ }^{34-37}$ Data on ultrastructural changes after barrier-protective signaling are less abundant. ${ }^{38}$ Therefore, in the present study, we chose a comprehensive approach correlating biochemical, functional, and morphological changes of the endothelial barrier to pinpoint functionally relevant effects of CAMP and Rac1 signaling on structural properties of endothelial contact zones. An increase of cAMP levels by F/R and Epac/Rap1 signaling by O-Me-cAMP effectively enhanced barrier properties in microvascular endothelium in vivo and in vitro, whereas no changes were detectable in macrovascular pulmonary artery endothelium. Under conditions of barrier stabilization, VE-cadherin and claudin 5 immunostainings were rearranged in microvessels and in cultured cells derived from the microvasculature. Interference with Rac1 activation decreased barrier functions and effectively blocked stabilization and rearrangement of contact proteins mediated by Epac/Rap1 signaling. By ultrastructural analysis, we detected an increase of complex intercellular contact sites with prominent junctions under conditions of CAMP/Rac1-mediated enhancement of barrier properties. 


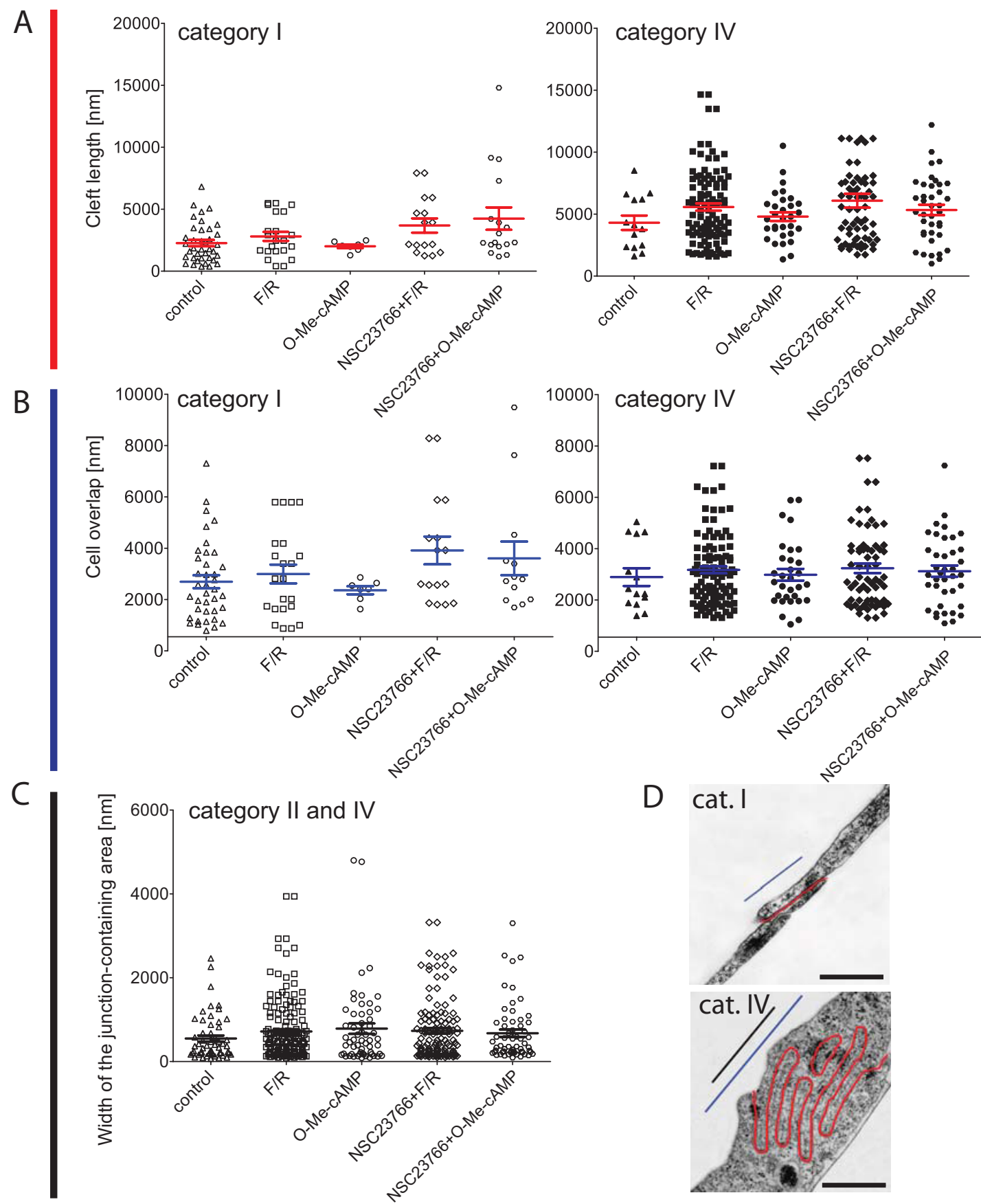

Figure 6. Refined ultrastructural analysis of intercellular contact zones of HDMECs. Detailed analysis of intercellular contact zones was performed, as depicted in $\mathbf{D}$ for categories 1 and 4 . Scale bars $=500 \mathrm{~nm}$. The length of the intercellular contact cleft (red line in $\mathbf{D}$ ) was evaluated and was substantially longer in category 4 compared with category 1 (A). The length of maximum cell overlap (blue line in D) was similar in both categories and did not significantly change under the various experimental conditions (B). The maximum width of the area enclosed by junctions (black line in $\mathbf{D}$ ) did not change by interference with cAMP/Racl signaling $(\mathbf{C}) . n=67$ to 123 junctions per condition.

\section{Barrier Enhancement by cAMP/Rac1 Apparently Is Mediated by Reorganization of Intercellular Contact Sites in Microvascular Endothelium}

In our study, a clear shift from simple overlapping cell contact sites without stabilizing intercellular junctions toward complex apicobasally interdigitating structures with numerous junctions was detectable by EM analysis under all conditions of increased barrier properties in microvas- cular cells. The data indicate that the complex contact zone phenotype represents the morphological correlate of increased endothelial barrier properties. This is further underscored by the fact that the contact cleft length was substantially longer in categories 3 and 4 compared with categories 1 and 2, which may limit the flux of water and solutes. ${ }^{24,39}$ Similarly, in placental microvascular endothelial cells, the cleft length appeared to be longer after long-term (48-hour) cAMP elevation, although there were 
different morphological characteristics of the contact zones. ${ }^{31}$ Because we detected a shift in the occurrence of category 1 contact sites without junctions to category 4 with junctions, in addition to the changes in the level of interdigitations, the absolute number of junctions, including adherens and tight junctions, was increased. This is in line with a study ${ }^{38}$ demonstrating increased tight junction strands in sections of microvessels after treatment with F/R (ie, under comparable conditions to those used in our study for in vivo and in vitro experiments). Interestingly, in perfused intact mesentery postcapillary venules, mostly simple overlapping contact structures, with or without junctions, were detected. ${ }^{38}$ In addition, we found contact zones displaying the complex interdigitating morphological features in vessels perfused under control conditions. It will be a subject of further investigations whether CAMP elevation changes the frequency of the complex contact morphological features in our experimental in vivo setup.

Cultured endothelial monolayers in vitro only partially reflect the situation in vivo because physiological pressure, blood flow, and cells such as pericytes are absent. ${ }^{40}$ Thus, it is not surprising that baseline permeability is higher in cultured cells compared with intact microvessels. ${ }^{2}$ This notion underscores the necessity of comparative in vivo and in vitro studies regarding the relevance of specific mechanisms. Therefore, we confirmed that increased CAMP under conditions used for the ultrastructural analysis in vitro was sufficient to reduce $L_{p}$ and to cause rearrangement of adherens junctions indicative for barrier enhancement in intact microvessels in vivo.

In parallel with increased intercellular contact zone complexity, we detected an augmented staining intensity and a reduction of mean width of the area containing tight junction marker claudin 5 and adherens junction protein VE-cadherin at cell borders in response to cAMP/Rac1 signaling. Such linearization and increase in signal strength were commonly observed after modulation of cAMP levels in endothelial cells. ${ }^{9,14,41,42}$ The increase in staining intensity may well be explained by the higher numbers of junctions observed by our EM analysis and, thus, likely reflects the reorganization of intercellular contact sites after augmented CAMP signaling. In addition, we more closely analyzed intercellular contact zones by EM. However, no differences regarding cleft length or cell overlap within one category were observed. In immunostaining experiments, the mean width of the VE-cadherin or claudin 5-positive zone varied between $2.4 \mu \mathrm{m}$ and $8.3 \mu \mathrm{m}$, depending on the experimental condition, whereas the width of the junction containing contact zone was stable at approximately $0.7 \mu \mathrm{m}$, as detected by EM. Thus, the reduction of immunostaining width may not be attributed to simple narrowing of the cell contact area. Rather, cell shape seems to be altered because VEcadherin and claudin 5-positive structures running perpendicular to cell borders were strongly reduced after cAMP/Rac1 signaling, resulting in a linearized appearance of cell borders. These orthogonal lines may be highly mobile adherens and tight junction-protein-positive cell processes observed by others, ${ }^{25}$ which may be reduced under conditions of increased CAMP/Rac1 sig- naling and, thus, may account for the narrowed immunostaining-positive zone. Therefore, after cAMP increase, the cell monolayer may undergo a change from interdigitations in the $x$-y (horizontal) plane to interdigitations in the $x-z$ (vertical) plane. This concept may also help to answer the important question of where the necessary membrane is derived from for the enhanced interdigitations in the $x-z$ plane in response to cAMP increase. Because no obvious changes in the morphological features of the rest of the endothelial cell were detectable, it is possible that these extensive membrane reorganizations are confined to the cell contact zones. This may be related to the increased occurrence of cortical actin filaments after cAMP enhancement (Figure 3).

This study focuses on changes of the paracellular pathway of fluid and solute exchange because this is a highly important pathway altered under acute inflammatory conditions. In addition to the paracellular pathway, a transcellular pathway exists that mainly contributes to the transport of macromolecules, such as albumin, and in which caveolae and vesiculovacuolar organelles play major roles. ${ }^{4,43}$ It cannot be excluded that CAMP changes also influence transcellular permeability; however, to our knowledge, no direct influence of cAMP signaling has been demonstrated within the endothelium. Nevertheless, CAMP signaling components are enriched in caveolae; and regulatory proteins of caveolae dynamics, such as Src kinases, are targets within the CAMP network, at least in nonendothelial cells. ${ }^{44-47}$ Therefore, a regulation may be conceivable.

\section{Role of Rac1 in CAMP-Mediated Barrier Stabilization}

CAMP signaling increases Rac1 activity. ${ }^{6}$ In this study, both activation of Epac/Rap1 signaling by O-Me-cAMP and an F/R-mediated cAMP increase caused Rac1 activation, augmented barrier functions, and changed contact morphological features to the more complex phenotype. On the other hand, interference with Rac1 activation by NSC-23766 destabilized endothelial barrier properties. The latter observation demonstrates that a constant activation of Rac1 is necessary to maintain baseline barrier stability. In addition, NSC-23766 completely prevented O-Me-cAMP-induced barrier stabilization and Rac1 activation. In contrast, F/R after NSC-23766 treatment rescued barrier function and morphological features, at least to control levels, although Rac1 activity was blunted under this condition. This indicates that Epac/Rap1-mediated strengthening of the endothelial barrier by O-Me-cAMP is strictly dependent on Rac1 activation by GTP-exchange factor Tiam or Trio; in contrast, increased cAMP in response to $F / R$, presumably via protein kinase A, may exert this effect, at least in part, by mechanisms other than Rac1 activation. This can be concluded from the fact that $F / R$ blocked barrier compromise induced by NSC-23766 in TER measurements under conditions in which Rac1 activation was rather weak. GTPases other than Rac1 may account for the rescue of barrier properties; however, our data indicate that at least Cdc42 is not involved. Nevertheless, the data further support the proposition that 
changes in endothelial contact zone architecture are one common effector of all barrier-strengthening pathways.

\section{cAMP Signaling Is Barrier Protective via Rac1 in Microvascular HDMECs but Not in Macrovascular PAECs}

In this study, we aimed to compare the effects of cAMP and Rac1 on contact zone reorganization in both microvascular and macrovascular endothelium. Surprisingly, we detected barrier stabilization and morphological reorganization on the level of light microscopy and EM by F/R or O-Me-cAMP in microvascular HDMECs only. Further biochemical analysis of PAEC monolayers revealed that F/R treatment under the conditions used was sufficient to increase cAMP levels but was similar to O-Me-cAMP, ineffective to cause activation of Rac1. Therefore, we assume that ineffective activation of Rac1 prevented cAMP-mediated barrier stabilization that, again, points to a central role of Rac1 in regulating the endothelial barrier. In addition, this aspect may represent a critical difference between microvascular and macrovascular endothelial cells in general or at least in macrovascular cells from a specific part of the vascular tree, such as the pulmonary artery. Of course, the differences observed between HDMECs and PAECs in response to a cAMP increase may also have other causes, such as species differences or culture conditions. Nevertheless, inflammatory reactions are known to primarily affect postcapillary venules, whereas the effects on the macrovasculature are negligible. ${ }^{3}$ According to our data, differences in CAMPmediated Rac1 regulation may contribute to this phenomenon. However, more studies to characterize cAMP/Rac1 signaling effects in vivo are required to fully understand these observations.

\section{Acknowledgments}

We thank Detlev Drenckhahn, M.D., and Nicolas Schlegel, M.D., for helpful discussions and technical advice; Yvonne Baumer, Ph.D., for providing antibody reagents; and Lisa Bergauer, Tetjana Frantzeskakis, Nadja Niedermeier, Karin Reinfurt-Gehm, and Sieglinde Schenk for skillful technical assistance.

\section{References}

1. Dejana E, Tournier-Lasserve E, Weinstein BM: The control of vascular integrity by endothelial cell junctions: molecular basis and pathological implications. Dev Cell 2009, 16:209-221

2. Michel CC, Curry FE: Microvascular permeability. Physiol Rev 1999, 79:703-761

3. Aird WC: Phenotypic heterogeneity of the endothelium, I: structure, function, and mechanisms. Circ Res 2007, 100:158-173

4. Mehta D, Malik AB: Signaling mechanisms regulating endothelial permeability. Physiol Rev 2006, 86:279-367

5. Franke WW: Discovering the molecular components of intercellular junctions: a historical view. Cold Spring Harb Perspect Biol 2009, 1:a003061

6. Spindler V, Schlegel N, Waschke J: Role of GTPases in control of microvascular permeability. Cardiovasc Res 2010, 87:243-253
7. Beckers CM, van Hinsbergh VW, van Nieuw Amerongen GP: Driving Rho GTPase activity in endothelial cells regulates barrier integrity. Thromb Haemost 2010, 103:40-55

8. Birukova AA, Burdette D, Moldobaeva N, Xing J, Fu P, Birukov KG Rac GTPase is a hub for protein kinase $A$ and Epac signaling in endothelial barrier protection by cAMP. Microvasc Res 2010, 79:128-138

9. Cullere X, Shaw SK, Andersson L, Hirahashi J, Luscinskas FW, Mayadas TN: Regulation of vascular endothelial barrier function by Epac, a cAMP-activated exchange factor for Rap GTPase. Blood 2005, 105 : 1950-1955

10. Fukuhara S, Sakurai A, Sano H, Yamagishi A, Somekawa S, Takakura N, Saito Y, Kangawa K, Mochizuki N: Cyclic AMP potentiates vascular endothelial cadherin-mediated cell-cell contact to enhance endothelial barrier function through an Epac-Rap1 signaling pathway. Mol Cell Biol 2005, 25:136-146

11. Schlegel N, Waschke J: Vasodilator-stimulated phosphoprotein: crucial for activation of Rac1 in endothelial barrier maintenance. Cardiovasc Res 2010, 81:1-3

12. Schlegel N, Baumer Y, Drenckhahn D, Waschke J: Lipopolysaccharide-induced endothelial barrier breakdown is cyclic adenosine monophosphate dependent in vivo and in vitro. Crit Care Med 2009, 37:1735-1743

13. Schlegel N, Waschke J: Impaired cAMP and Rac 1 signaling contribute to TNF-alpha-induced endothelial barrier breakdown in microvascular endothelium. Microcirculation 2009, 16:521-533

14. Baumer Y, Spindler V, Werthmann RC, Bunemann M, Waschke J: Role of Rac 1 and CAMP in endothelial barrier stabilization and thrombininduced barrier breakdown. J Cell Physiol 2009, 220:716-726

15. Adamson RH, Curry FR: Rapid calcium-dependent reduction of intraendothelial CAMP: a trigger to increase vascular permeability? J Physiol 2009, 587(Pt 16):3975

16. Baumer Y, Burger S, Curry FE, Golenhofen N, Drenckhahn D, Waschke J: Differential role of Rho GTPases in endothelial barrier regulation dependent on endothelial cell origin. Histochem Cell Biol 2008, 129:179-191

17. Golenhofen N, Ness W, Wawrousek EF, Drenckhahn D: Expression and induction of the stress protein alpha-B-crystallin in vascular endothelial cells. Histochem Cell Biol 2002, 117:203-209

18. Waschke J, Baumgartner W, Adamson RH, Zeng M, Aktories K, Barth $\mathrm{H}$, Wilde C, Curry FE, Drenckhahn D: Requirement of Rac activity for maintenance of capillary endothelial barrier properties. Am J Physiol Heart Circ Physiol 2004, 286:H394-H401

19. Schnittler HJ, Wilke A, Gress T, Suttorp N, Drenckhahn D: Role of actin and myosin in the control of paracellular permeability in pig, rat and human vascular endothelium. J Physiol 1990, 431:379-401

20. Gao Y, Dickerson JB, Guo F, Zheng J, Zheng Y: Rational design and characterization of a Rac GTPase-specific small molecule inhibitor. Proc Natl Acad Sci U S A 2004, 101:7618-7623

21. Michel CC, Mason JC, Curry FE, Tooke JE, Hunter PJ: A development of the Landis technique for measuring the filtration coefficient of individual capillaries in the frog mesentery. Exp Physiol 1974, 59:283-309

22. Wijsman J, Shivers R: Immortalized mouse brain endothelial cells are ultrastructurally similar to endothelial cells and respond to astrocyteconditioned medium. In Vitro Cell Dev Biol Anim 1998, 34:777-784

23. Reynolds ES: The use of lead citrate at high $\mathrm{pH}$ as an electronopaque stain in electron microscopy. J Cell Biol 1963, 17:208-212

24. Adamson $\mathrm{RH}$, Michel $\mathrm{CC}$ : Pathways through the intercellular clefts of frog mesenteric capillaries. J Physiol 1993, 466:303-327

25. Millán J, Cain RJ, Reglero-Real N, Bigarella C, Marcos-Ramiro B, Fernández-Martín L, Correas I, Ridley AJ: Adherens junctions connect stress fibres between adjacent endothelial cells. BMC Biol 2010, 8:11

26. Ruffer C, Strey A, Janning A, Kim KS, Gerke V: Cell-cell junctions of dermal microvascular endothelial cells contain tight and adherens junction proteins in spatial proximity. Biochemistry 2004, 43:5360-5369

27. Drenckhahn D, Ness W: The Endothelial Contractile Cytoskeleton. Stuttgart, Schattauer, 1997, pp 1-25

28. Gustafsson F, Mikkelsen HB, Arensbak B, Thuneberg L, Neve S, Jensen LJ, Holstein-Rathlou N-H: Expression of connexin 37, 40 and 43 in rat mesenteric arterioles and resistance arteries. Histochem Cell Biol 2003, 119:139-148

29. Simionescu M, Simionescu N, Palade GE: Segmental differentiations of cell junctions in the vascular endothelium: the microvasculature. J Cell Biol 1975, 67:863-885 
30. Hermanns MI, Unger RE, Kehe K, Peters K, Kirkpatrick CJ: Lung epithelial cell lines in coculture with human pulmonary microvascular endothelial cells: development of an alveolo-capillary barrier in vitro. Lab Invest 2004, 84:736-752

31. Dye JF, Leach L, Clark P, Firth JA: Cyclic AMP and acidic fibroblast growth factor have opposing effects on tight and adherens junctions in microvascular endothelial cells in vitro. Microvasc Res 2001, 62:94-113

32. Adamson RH, Lenz JF, Zhang X, Adamson GN, Weinbaum S, Curry FE: Oncotic pressures opposing filtration across non-fenestrated rat microvessels. J Physiol 2004, 557:889-907

33. King J, Hamil T, Creighton J, Wu S, Bhat P, McDonald F, Stevens T: Structural and functional characteristics of lung macro- and microvascular endothelial cell phenotypes. Microvasc Res 2004, 67:139-151

34. Majno G, Palade GE: Studies on inflammation, 1: the effect of histamine and serotonin on vascular permeability: an electron microscopic study. J Biophys Biochem Cytol 1961, 11:571-605

35. Hirata A, Baluk P, Fujiwara T, McDonald DM: Location of focal silver staining at endothelial gaps in inflamed venules examined by scanning electron microscopy. Am J Physiol Lung Cell Mol Physiol 1995, 269:L403-L418

36. Baluk P, Hirata A, Thurston G, Fujiwara T, Neal CR, Michel CC, McDonald DM: Endothelial gaps: time course of formation and closure in inflamed venules of rats. Am J Physiol Lung Cell Mol Physiol 1997, 272:L155-L170

37. Knezevic II, Predescu SA, Neamu RF, Gorovoy MS, Knezevic NM, Easington C, Malik AB, Predescu DN: Tiam1 and Rac1 are required for platelet-activating factor-induced endothelial junctional disassembly and increase in vascular permeability. J Biol Chem 2009, 284:5381-5394

38. Adamson RH, Liu B, Fry GN, Rubin LL, Curry FE: Microvascular permeability and number of tight junctions are modulated by CAMP. Am J Physiol Heart Circ Physiol 1998, 274:H1885-H1894
39. Clough G, Michel CC: Quantitative comparisons of hydraulic permeability and endothelial intercellular cleft dimensions in single frog capillaries. J Physiol 1988, 405:563-576

40. Curry F-RE, Adamson RH: Vascular permeability modulation at the cell, microvessel, or whole organ level: towards closing gaps in our knowledge. Cardiovasc Res 2010, 87:218-229

41. Kooistra MRH, Corada M, Dejana E, Bos JL: Epac1 regulates integrity of endothelial cell junctions through VE-cadherin. FEBS Lett 2005, 579:4966-4972

42. Baumer Y, Drenckhahn D, Waschke J: cAMP induced Rac 1-mediated cytoskeletal reorganization in microvascular endothelium. Histochem Cell Biol 2008, 129:765-778

43. Feng D, Nagy JA, Hipp J, Dvorak HF, Dvorak AM: Vesiculo-vacuolar organelles and the regulation of venule permeability to macromolecules by vascular permeability factor, histamine, and serotonin. J Exp Med 1996, 183:1981-1986

44. Razani B, Lisanti MP: Two distinct caveolin-1 domains mediate the functional interaction of caveolin-1 with protein kinase A. Am J Physiol Cell Physiol 281:C1241-C1250

45. Vang T, Torgersen KM, Sundvold V, Saxena M, Levy FO, Skålhegg BS, Hansson V, Mustelin T, Taskén K: Activation of the $\mathrm{COOH}$ terminal Src kinase (Csk) by cAMP-dependent protein kinase inhibits signaling through the T cell receptor. J Exp Med 2001, 193:497-507

46. Gates A, Hohenester S, Anwer MS, Webster CRL: cAMP-GEF cytoprotection by Src tyrosine kinase activation of phosphoinositide-3kinase p110 beta/alpha in rat hepatocytes. Am J Physiol Gastrointest Liver Physiol 2009, 296:G764-G774

47. Schwencke C, Yamamoto M, Okumura S, Toya Y, Kim S-J, Ishikawa Y: Compartmentation of cyclic adenosine $3^{\prime}, 5^{\prime}$-monophosphate signaling in caveolae. Mol Endocrinol 1999, 13:1061-1070 